\title{
RESULTS OF SURGICAL MANAGMENT OF PRIMARY MITRAL REGURGITATION IN A SINGLE-CENTER STUDY
}

\author{
Oleksiy Myshakivskyy \\ Cardiac surgeon \\ Department of cardiac surgery \\ Lviv Regional Clinical Hospital \\ 7 Chernihivska str., Lviv, Ukraine, 79000 \\ Danylo Halytsky Lviv National Medical University \\ 69 Pekarska str., Lviv, Ukraine, 79010 \\ oleksijmy@gmail.com
}

\begin{abstract}
Mitral regurgitation (MR) remains the second dominant defect in the structure of valvular cardiac diseases.

It affects more than 2 million people in the USA. Basic causes are classified as degenerative (with valve prolapse) and ischemic (due to ischemic heart disease) in advanced countries or rheumatic ones (in developing countries).

Alone radical method of MR treatment is surgical correction through mitral valve repair (MVRe) or replacement (MVR) yielding definitely higher survival percentage and improvement of heart failure (HF) class comparing to pharmacotherapy.

Evolution of approaches to the management of non-ischemic MR passed through some stages starting from predominantly MVR to organ-preserving approaches like plastic repair.

In the prospective single-center study were analyzed the results of treatment of 72 patients with primary MR (PMR) who were subjected to mitral valve replacement (MVR) or plastic mitral valve repair (MVRe) performed in the Department of cardiac surgery affiliated with Lviv regional clinical hospital (Ukraine) since October, 2013 till February, 2016.

The conclusions of performed study are next:

1. Key direct cause of MR is the chordal rupture of MV cusps; etiological factor in the majority of advanced countries is degenerative changes in contrast to rheumatic changes in the developing countries.

2. Principal method of MR surgical correction in out center is MVR, though the preferable global trend is MVRe.

3. Complications and lethality percentages in this study were higher among the patients from MVR group. Improvement of HF class according to NYHA was more evident in the MVRe group.

This corresponds to results of other studies and guidelines that recommend MVRe as optional method for MR correction.

Keywords: primary mitral regurgitation, mitral valve replacement, mitral valve repair, tricuspid valve repair, heart failure, effective ejection fraction.
\end{abstract}

\section{Introduction}

According to "The Euro Heart Survey on Valvular Heart Disease", published in 2003, mitral regurgitation (MR) is the second frequent valvular defect after aortic valve stenosis [1].

The majority of MR causes in advanced countries are degenerative valvular changes and ischemic MR in contrast to developing countries, where rheumatic causes are dominant [2, 3].

Drug therapy as single method leads to $3 \%$ and over $6 \%$ of annual mortality for patients with moderate and severe MR accordantly [2].

There is only one radical method of MR treatment - surgical correction. It may be mitral valve replacement (MVR) with prosthesis valve, or mitral valve repair (MVRe). Surgery improves patients surviving percentage and heart failure (HF) class unlike drug therapy [4].

Plastic repair of MV is preferable to MVR in modern valve surgery era [5, 6], because of lower operative mortality comparing to MVR [7], no need of anticoagulation therapy after short "post-surgery" time [8], lower infective complications incidence associated with valve surgery [9].

\section{Aim of the research}

Aim of this study is prospective assessment of the features or discrepancies referring to the results of surgical management of the patients with primary mitral regurgitation (MR). 


\section{Materials and methods}

In the prospective single-center study are the results of treatment among 72 patients with primary MR, who were subjected to MVR or plastic repair in the Department of cardiac surgery affiliated with Lviv regional clinical hospital since October, 2013 till February, 2016.

Registration of all parameters was performed 3 times (pre-op, early post-op period and in 3 months after the operation) using special patient's visit registration form.

The stages of MR and TR were defined - mild, moderate and severe ones. Therefore, have been determined a ratio between regurgitation jet area and area of left atrium, vena contract index and PISA method.

Echocardiography was performed before the operation, in 5-7 days and in 3 months after the operation. Echocardiograph Sonos 5500 (Phillips) was used with multifrequency cardiac probe $(1-2.5 \mathrm{MHz})$.

Besides ejection fraction (EF) was also provided measurement using Simpson method and Teicholz method so-called "effective" (Doppler-derived) EF of LV. The essence of this method is based upon the fact that LV stroke volume (SV) is determined with Doppler method and LV end-diastolic volume (EDV) is determined with Teicholz method. EF is calculated by the formula LV SV/EDV.

Statistical analysis was accomplished with an advanced analytics software package "Statistica for Windows 5.0". Because the studied groups were not equal and parametric variables distributions were not normal (according to Shapiro-Wilk' W test), they were described with median (lower-upper quartiles). Between-group differences were evaluated using Mann-Whitney U test for continuous and ranged variables or Fisher's exact test for categorical variables. For qualitative parameters' (frequency tables) comparison $\chi^{2}$-test was used. Difference between parameters was significant when $\mathrm{p}<0.05$.

\section{Results}

Males prevailed in the MVRe group - 12 persons (66.67\%), female prevailed in the MVR group - 29 persons (53.7\%). Age median (minimum-maximum) was 58 years (19-79 years).

The most common etiological factor of MR was MV degenerative changes - 41 patients (56.9\%). The most often direct cause of MR was chordal rupture - 43 cases (59.7 \%) (Table 1).

In all patients surgical access was performed through median sternotomy. After the full heparinization heart-lung machine was connected through cannulation of ascending aorta and both vena cava separately. Moderate hypothermia $\left(28{ }^{\circ} \mathrm{C}\right)$ and cold potassium-blood cardioplegy were used. Applied approaches to the MV were trans-septal one and through the left atrium.

Plastic repair of MV was done in 18 patients (25\%), MVR was performed in 54 patients $(75 \%)$.

In the MVRe group before the operation among all patients moderate MR was revealed 19 persons $(100 \%)$.

In the MVR group before the operation moderate MR also was revealed - 54 patients $(100 \%)$.

On the $1^{\text {st }}$ and $2^{\text {nd }}$ control post-op examinations in the MVRe group MR was absent among 16 patients $(88.89 \%)$, only 2 patients $(11.11 \%$ ) had mild MR.

On the $1^{\text {st }}$ and $2^{\text {nd }}$ control post-op examinations in the MVR group MR was absent among all 51 patients (100\%).

In the MVR group predominantly two cusps were affected -20 patients (35\%), in the MVRe group posterolateral cusp was involved - 11 patients $(23.5 \%)$.

Plastic repair with usage of annuloplasty ring was performed among 17 patients; plastic repair without annuloplasty ring was performed only in 1 patient. Plastic rings produced by "Carpentier Edwards" (n=3 [17.6 \%]) and "St. Jude" (n=14 [82.4]) have been used. Rings" sizes were $36 \mathrm{~mm}(\mathrm{n}=1[5.88 \%]) ; 34 \mathrm{~mm}(\mathrm{n}=1$ [5.88\%]); $32 \mathrm{~mm}(\mathrm{n}=6$ [35.29\%]); $30 \mathrm{~mm}(\mathrm{n}=7$ [41.18\%]); $29 \mathrm{~mm}(\mathrm{n}=1$ [5.88 \%]); $28 \mathrm{~mm}(\mathrm{n}=1$ [5.88 \%]). For neochords' preparation Gore-Tex and Ethibond have been used. Types of MV plastic correction are presented in the Table 2. 
Table 1

Patients' distribution according to gender, etiological and direct causes of mitral regurgitation $(n=72)$

\begin{tabular}{cc}
\hline Parameter & Number (\%) \\
Males & $37(51.4)$ \\
Females & $35(48.6)$ \\
Etiological factor of MR & $72(100)$ \\
degenerative changes in MV & $41(56.9)$ \\
chordal rupture in visually unchanged cusps & $11(15.3)$ \\
infective endocarditis & $15(20.8)$ \\
rheumatic changes in MV & $4(5.6)$ \\
isolated prolapse of cusps & $1(1.4)$ \\
Direct cause of MR & $72(113.8)$ \\
chordal rupture & $43(59.7)$ \\
prolapse of cusps & $7(9.7)$ \\
vegetations & $12(16.7)$ \\
leaflet perforation & $5(6.9)$ \\
diffuse changes & $15(20.8)$ \\
Combination of two causes & 10
\end{tabular}

Table 2

Types of MV plastic corrections (in some cases combination of several types was simultaneously performed)

\begin{tabular}{cc}
\hline Type of plastic & Number of patients \\
\hline Linear suturing of comissura & 1 \\
Wedge resection & 7 \\
Repair with usage of only annuloplasty ring & 1 \\
MV repair using Alfieri stitch & 1 \\
Neochords' implantation & 3 \\
Segment's plication without resection in case of segmental prolaps & 2 \\
Linear suturing of the defect without annuloplasty ring & 1 \\
Sectoral resection (not specified in the protocol) & 3
\end{tabular}

Among 4 patients performed MVRe did not yield satisfactory result during Hydraulic test, so they immediately were subjected to MVR. During the statistical analysis these patients were included into MVR group.

Among the MV prostheses most often ones produced by "St. Jude" - 36 cases $(70.6 \%)$, "ATS" - 8 patients (15.7\%), and other companies ("Medtronic", "Carbomedics", "Bjork") - 7 patients have been used. In 1 patient bioprosthesis St. Jude Epic $(29 \mathrm{~mm})$ was implanted.

Sizes of the used prostheses were $33 \mathrm{~mm}(\mathrm{n}=1(1.85 \%)) ; 31 \mathrm{~mm}(\mathrm{n}=1(1.85 \%)) ; 29 \mathrm{~mm}$ $(\mathrm{n}=20$ (38.89)); $27 \mathrm{~mm}(\mathrm{n}=19(38.89 \%)) ; 26 \mathrm{~mm}(\mathrm{n}=2(3.70)) ; 25 \mathrm{~mm}(\mathrm{n}=8(14.81 \%))$.

De Vega tricuspid valve repair was performed among 60 patients $(83.3 \%)$ (1 patient required additional suturing of TV cusp), among 12 patients $(16.7 \%)$ this approach was not used. 
Next concomitant procedures at the time of mitral valve repair were performed: 1 coronary artery bypass surgery and 1 PFO closure.

Signs of chronic heart failure (according to NYHA classification) were observed among all 72 pre-op patients.

Among pre-op patients subjected to MVR HF class 2 was dominant - 10 patients $(55.56 \%)$.

After the intervention on the $1^{\text {st }}$ control examination in MVR group 9 patients $(50 \%)$ had HF class 1 and 2.

On the $2^{\text {nd }}$ control examination dominated patients with HF class $1-14$ persons $(77.78 \%)$, 2 patients (11.11\%) had HF class 2 and 2 patients (11.11\%) had HF class 0 according to NYHA.

Among pre-op patients from MVR group dominated HF class 3 - 38 persons $(70.37 \%)$.

After the operation on the $1^{\text {st }}$ control examination among the patients subjected to MVR dominated persons with HF class $2-40$ patients (74.07\%); also 9 patients (16.67\%) had HF class 3 and 5 patients $(9.26 \%)$ had HF class 1 .

After the operation on the $2^{\text {nd }}$ control examination in this group of patients 29 persons (56.86 \%) had HF class 2, 2 persons (3.92 \%) had HF class 3 and 20 persons $(39.22 \%)$ had HF class 1 (Table 3).

Table 3

Functional class according to NYHA

\begin{tabular}{|c|c|c|c|c|c|c|}
\hline Index & Pre-op (\%) & $\begin{array}{c}1^{\text {st }} \text { post-op } \\
\text { examination }(\%)\end{array}$ & $\begin{array}{c}2^{\text {nd }} \text { post-op } \\
\text { examination (\%) }\end{array}$ & $p_{1-2}$ & $\mathbf{p}_{2-3}$ & $P_{1-3}$ \\
\hline NYHA 0 & 0 & 0 & $2(2.9)$ & & & \\
\hline 1 & $2(2.8)$ & 14 (19.4) & 34 (49.3) & & & \\
\hline 2 & $21(29.2)$ & $49(68.1)$ & $31(44.9)$ & $<0.001$ & $<0.001$ & $<0.001$ \\
\hline 3 & $43(59.7)$ & $9(12.5)$ & $2(2.9)$ & & & \\
\hline 4 & $6(8.3)$ & 0 & 0 & & & \\
\hline In total & 72 & 72 & 69 & & & \\
\hline
\end{tabular}

Positive trend referring to restoration of normal cardiac rhythm after the surgical intervention was observed only among the patients subjected to MVRe: 14 pre-op persons $(77.78 \%$ ) and 16 persons $(88.89 \%)$ on the $1^{\text {st }}$ and $2^{\text {nd }}$ pos-op examination had sinus rhythm.

Negative trend referring to restoration of normal cardiac rhythm after the surgical intervention was observed in the group of patients exposed to MVR: 32 pre-op patients (59.26\%), 31 patients $(57.42 \%)$ on the $1^{\text {st }}$ post op examination and 29 patients $(56.86 \%)$ on the $2^{\text {nd }}$ post-op one had sinus rhythm.

Absolute majority of pre-op patients had left ventricular dilatation (LVD), increase of LV and RV end-diastolic volume (EDV) and significant increase of pulmonary artery (PA) systolic pressure. These parameters clearly diminished in several days after the operation and such a dynamics for some indices (size of right atrium [RA], RV plus PA systolic pressure) and statistically significant improvement of mentioned above echocardiographic post-op parameters persisted up to $2^{\text {nd }}$ post-op examination that testifies about positive remodeling after surgery.

EF measured with Simpson method and Teicholz method has decreased in the early postop period and subsequently did not significantly change; some increase of EF measured with Simpson method on the $3^{\text {rd }}$ examination comparing to the $2^{\text {nd }}$ one have been noted. In contrast, the pre-op effective (Doppler-derived) EF was significantly decreased comparing to normal parameters, clearly has increased in the early post-op period and has been reliably increasing up to the $3^{\text {rd }}$ examination (Table 4).

In the MVR group one complication (acute dynamic ileus) occurred, that required laparotomy and small intestine's decompression. 
Table 4

Pre-op and post-op echocardiographic indices, median (min.-max.), quartiles [upper-lower]; ESV - endsystolic volume of LV, EF - ejection fraction

\begin{tabular}{|c|c|c|c|c|c|c|}
\hline Parameter & Pre-op & $\begin{array}{c}1^{\text {st }} \text { post-op } \\
\text { examination }\end{array}$ & $\begin{array}{c}2^{\text {nd }} \text { post-op } \\
\text { examination }\end{array}$ & $p_{1-2}$ & $\mathbf{p}_{2-3}$ & $\mathbf{P}_{1-3}$ \\
\hline EDV & $5.35(4.3-6.9)$ & $4.65(3.8-6.6)$ & $4.6(3.8-6.3)$ & $<0.0001$ & 0.31 & $<0.0001$ \\
\hline ESV & $\begin{array}{c}3.25(2.4-4.9) \\
{[3.1-3.7]}\end{array}$ & $\begin{array}{c}3.25(2.2-5) \\
{[2.8-3.8]}\end{array}$ & $3.1(2.1-4.8)$ & 0,56 & 0.25 & 0.16 \\
\hline LA & $5.1(4-8 ., 8)[4.7-5.55]$ & $4.6(3-8.2)[4.2-4.9]$ & $\begin{array}{c}4.4(3.4-7.7) \\
{[4.1-4.9]}\end{array}$ & $<0.0001$ & 0.038 & $<0.0001$ \\
\hline LV & $3(2.1-3.9)[2.8-3.1]$ & $\begin{array}{c}\text { 2. } 8(1.8-3.8) \\
{[2.6-3]}\end{array}$ & $2.6(2-3.6)[2.4-2.9]$ & $<0.0001$ & 0.01 & $<0.0001$ \\
\hline PA systolic pressure & $57(0-105)[47.5-66.5]$ & $38(0-70)[30.5-44]$ & $34(0-70)$ [29-39] & $<0.0001$ & $<0.0001$ & $<0.0001$ \\
\hline EF (Teicholz method) & $68(50-82)[63.5-71]$ & $58(30-76)[47-65]$ & $57(24-77)$ [52-61] & $<0.0001$ & 0.97 & $<0.0001$ \\
\hline EF (Simpson method) & $69(50-72)[68-71]$ & $58(23-68)[53-61]$ & $60(26-66)[58-62]$ & $<0.0001$ & 0.016 & $<0.0001$ \\
\hline Effective EF & $36(17-77)[30-41]$ & $58(23-87)[50-69]$ & $64(26-84)[57-75]$ & $<0.0001$ & 0.009 & $<0.0001$ \\
\hline
\end{tabular}

In the MVR next complications were observed: pericardial drainage (1 patient); two successive pericardial drainages (1 patient); auxiliary artery embolism with subsequent thrombectomy (1 patient); acute ischemic stroke (1 patient).

In the MVR group after the operation 3 persons died: one female patient died on the $60^{\text {th }}$ day after the surgery (infectious endocarditis with embologenic vegetations of the MV posterolateral cusp and abscess of the LV posterior wall) due to polyorganic (in particular, renal) insufficiency; one male patient (pre-op infectious endocarditis with vegetations and perforation of MV anteromedial cusp); one male patient died due to sudden cardiac arrest with subsequent failed cardiopulmonary resuscitation.

In the group of patients subjected to MVRe due to primary MR during in-patient department stay and next two control visits no lethal cases were revealed.

\section{Discussion}

MVRe in compare to MVR yields better results and among patients with severe organic MR diminished mortality down to $70 \%$. The best short-term and long-term results are registered in non-symptomatic patients operated in big referential centers with low surgical mortality (less than $1 \%$ ) and high percentage of repairs (over 80-90\%). These results stress the importance of early detection, assessment and management of MR [2]. MVR or MVRe must be done in a timely fashion before the onset of symptoms, ventricular dysfunction or atrial fibrillation [5], to achive best results. Especially atrial fibrillation is negative precursor for higher mortality rates, risk of stroke and left ventricular dysfunction in patients with MR [10].

MVRe has also benefits because of no need of long aggressive anticoagulation therapy [11].

Long-term studies showed that comparing to medical treatment early surgical intervention on MV among patients with prolapse of cusps was associated with significantly lower risk of the future death due to cardiac causes or HF and longer life expectancy [4]. Also bleeding risk in older patients is lower when MVR performed [12].

Echocardiographic methods of MR severity assessment give a chance to reveal promptly disease's progression before the appearance of the evident or new symptoms. In turn, it allows to specify the timely indications for surgical intervention during repeated examinations aimed at regurgitation's stage assessment $[13,14]$. 
In the study conducted by James et al. [15] it was proved, that during 8 years among 58370 patients subjected to isolated primary operations on MV due to MR $(n=47.126)$ prevalence of MVRe comparing to MVR increased from $51 \%$ to $69 \%(\mathrm{p}<0.0001)$. Among the patients from MVR group ( $\mathrm{n}=24.404)$ evident decrease of mechanic prostheses' usage was observed: from $68 \%$ to $37 \%(\mathrm{p}<0.0001)$. Surgical mortality in MVR group was significantly higher comparing to MVRe $(3.8 \%$ versus $1.4 \%, \mathrm{p}<0.0001)$ that corresponds to this study results. Among the patients subjected to selective isolated MVRe $(\mathrm{n}=28.140)$ surgical mortality was $1.2 \%$. For non-symptomatic (class I) patients surgical mortality was $0.6 \%$.

By Catherine M. Otto [6] it was confirmed that surgical mortality is lower in MVRe comparing to MVR (2-4\% versus 5-10\%). Among the patients with MV prolapse remote clinical outcomes were wonderful, survival rate was 80 and $94 \%$ during the next 5 and 10 years in MVRe group comparing to 40 and $60 \%$ in MVR group. There trends correspond to the results of this study, characterized by lower mortality in MVRe group.

Gillinov et al. [3] noted, that in 10 years after the MVRe reoperation absence rate was $93 \%$. Among 30 patients, who required reoperations due to late valvular dysfunction, repair was unsuccessful in 16 persons (53\%) owing to progressive degenerative disease. Firmness/stability of the repair yielded negative result among the patients with preceding prolapse of posterolateral cusp, ones subjected to chordal shortening, independent annuloplasty, and resection of posterolateral cusp without annuloplasty. Results of the plastic correction were steadier in the patients with isolated prolapse of posterolateral cusp, exposed to resection of posterolateral cusp and annuloplasty. Independent (as the single method) chordal shortening, annuloplasty and resection of cusps without annuloplasty jeopardized positive remote results of surgical management.

Interestingly, according to some studies [16] surgical intervention was denied for $49 \%$ patients with severe symptomatic MR. Diminution of LV EF, old age, co-morbid conditions were the basic striking features of the patients who were denied. Importance of age and LV EF referring to patients' operability are still discussible.

Negative dynamics concerning restoration of sinus rhythm among the patients subjected to MVR probably may be explained by more severe preceding pre-op state, more prolonged medical history and consequently larger size of LA and prolonged medical history of atrial fibrillation. These factors are known negative predictors of preservation and restoration of sinus rhythm after the surgical correction of MR. Along with MV correction these patients were not exposed to simultaneous open-surgery isolation of pulmonary veins, ablation of caval-tricuspid isthmus and areas of possible arrhythmias expansion on the basis of MV [17]. Open ablation in atrial fibrillation improves post op outcome referring preservation of sinus rhythm and non-recurrence period's duration [18]. This procedure is feasible and safe $[19,20]$ in modern surgery era.

\section{Conclusions}

1. Key direct cause of MR is the chordal rupture of MV cusps; etiological factor in the majority of advanced countries is degenerative changes in contrast to rheumatic changes in the developing countries.

2. Principal method of MR surgical correction in out center is MVR, though the preferable global trend is MVRe.

3. Complications and lethality percentages in this study were higher among the patients from MVR group. Improvement of HF class according to NYHA was more evident in the MVRe group.

This corresponds to results of other studies and guidelines that recommend MVRe as optional method for MR correction.

\section{References}

[1] Iung, B. (2003). A prospective survey of patients with valvular heart disease in Europe: The Euro Heart Survey on Valvular Heart Disease. European Heart Journal, 24 (13), 1231-1243. doi: 10.1016/ s0195-668x(03)00201-x 
[2] Enriquez-Sarano, M., Akins, C. W., Vahanian, A. (2009). Mitral regurgitation. The Lancet, 373 (9672), 1382-1394. doi: 10.1016/s0140-6736(09)60692-9

[3] Gillinov, A. M., Cosgrove, D. M., Blackstone, E. H., Diaz, R., Arnold, J. H., Lytle, B. W. et. al. (1998). Durability of mitral valve repair for degenerative disease. The Journal of Thoracic and Cardiovascular Surgery, 116 (5), 734-743. doi: 10.1016/s0022-5223(98)00450-4

[4] Ling, L. H., Enriquez-Sarano, M., Seward, J. B., Orszulak, T. A., Schaff, H. V., Bailey, K. R. et. al. (1997). Early Surgery in Patients With Mitral Regurgitation Due to Flail Leaflets : A Long-term Outcome Study. Circulation, 96 (6), 1819-1825. doi: 10.1161/01.cir.96.6.1819

[5] Adams, D. H., Rosenhek, R., Falk, V. (2010). Degenerative mitral valve regurgitation: best practice revolution. European Heart Journal, 31 (16), 1958-1966. doi: 10.1093/eurheartj/ehq222

[6] Otto, C. M. (2001). Evaluation and Management of Chronic Mitral Regurgitation. New England Journal of Medicine, 345 (10), 740-746. doi: 10.1056/nejmcp003331

[7] Enriquez-Sarano, M., Basmadjian, A.-J., Rossi, A., Bailey, K. R., Seward, J. B., Tajik, A. J. (1999). Progression of mitral regurgitation. Journal of the American College of Cardiology, 34 (4), 1137-1144. doi: 10.1016/s0735-1097(99)00313-7

[8] Gammie, J. S., Sheng, S., Griffith, B. P., Peterson, E. D., Rankin, J. S., O’Brien, S. M., Brown, J. M. (2009). Trends in Mitral Valve Surgery in the United States: Results From The Society of Thoracic Surgeons Adult Cardiac Database. The Annals of Thoracic Surgery, 87 (5), 1431-1439. doi: 10.1016/ j.athoracsur.2009.01.064

[9] Mirabel, M., Iung, B., Baron, G., Messika-Zeitoun, D., Detaint, D., Vanoverschelde, J.-L. et. at. (2007). What are the characteristics of patients with severe, symptomatic, mitral regurgitation who are denied surgery? European Heart Journal, 28 (11), 1358-1365. doi: 10.1093/eurheartj/ehm001

[10] Lazopoulos, G., Mihas, C., Manns-Kantartzis, M., Kantartzis, M. (2013). Radiofrequency ablation for atrial fibrillation during concomitant cardiac surgery. Herz, 39 (2), 206-211. doi: 10.1007/s00059$013-3787-1$

[11] Dunning, J., Versteegh, M., Fabbri, A., Pavie, A., Kolh, P., Lockowandt, U., Nashef, S. A. M. (2008). Guideline on antiplatelet and anticoagulation management in cardiac surgery. European Journal of Cardio-Thoracic Surgery, 34 (1), 73-92. doi: 10.1016/j.ejcts.2008.02.024

[12] Fedoruk, L. M., Jamieson, W. R. E., Ling, H., MacNab, J. S., Germann, E., Karim, S. S., Lichtenstein, S. V. (2009). Predictors of recurrence and reoperation for prosthetic valve endocarditis after valve replacement surgery for native valve endocarditis. The Journal of Thoracic and Cardiovascular Surgery, 137 (2), 326-333. doi: 10.1016/j.jtcvs.2008.08.024

[13] Kang, D.-H., Kim, J. H., Rim, J. H., Kim, M.-J., Yun, S.-C., Song, J.-M. et. al. (2009). Comparison of Early Surgery Versus Conventional Treatment in Asymptomatic Severe Mitral Regurgitation. Circulation, 119 (6), 797-804. doi: 10.1161/circulationaha.108.802314

[14] Gammie, J. S., Sheng, S., Griffith, B. P., Peterson, E. D., Rankin, J. S., O’Brien, S. M., Brown, J. M. (2009). Trends in Mitral Valve Surgery in the United States: Results From The Society of Thoracic Surgeons Adult Cardiac Database. The Annals of Thoracic Surgery, 87 (5), 1431-1439. doi: 10.1016/ j.athoracsur.2009.01.064

[15] O’Gara, P., Sugeng, L., Lang, R., Sarano, M., Hung, J., Raman, S. et. al. (2008). The Role of Imaging in Chronic Degenerative Mitral Regurgitation. JACC: Cardiovascular Imaging, 1 (2), 221-237. doi: 10.1016/j.jcmg.2008.01.011

[16] Shuhaiber, J., Anderson, R. J. (2007). Meta-analysis of clinical outcomes following surgical mitral valve repair or replacement. European Journal of Cardio-Thoracic Surgery, 31 (2), 267-275. doi: 10.1016/ j.ejcts.2006.11.014

[17] Gillinov, A. M., Blackstone, E. H., Nowicki, E. R., Slisatkorn, W., Al-Dossari, G., Johnston, D. R. at. al. (2008). Valve repair versus valve replacement for degenerative mitral valve disease. The Journal of Thoracic and Cardiovascular Surgery, 135 (4), 885-893. doi: 10.1016/j.jtcvs.2007.11.039 
[18] Calkins, H., Brugada, J., Packer, D. L., Cappato, R., Chen, S.-A., Crijns, H. J. G. et. al. (2007). HRS/EHRA/ECAS expert consensus statement on catheter and surgical ablation of atrial fibrillation: recommendations for personnel, policy, procedures and follow-up. Europace, 9 (6), 335-379. doi: 10.1093/ europace/eum 120

[19] Pinho-Gomes, A. C., Amorim, M. J., Oliveira, S. M., Leite-Moreira, A. F. (2014). Surgical treatment of atrial fibrillation: an updated review. European Journal of Cardio-Thoracic Surgery, 46 (2), 167-178. doi: 10.1093/ejcts/ezt584

[20] Banach, M., Kourliouros, A., M. Reinhart, K., Benussi, S., P. Mikhailidis, D., Jahangiri, M. et. al. (2010). Postoperative Atrial Fibrillation - What Do We Really Know? Current Vascular Pharmacology, 8 (4), 553-572. doi: 10.2174/157016110791330807 